\title{
Complete Controllability of Impulsive Stochastic Integrodifferential Systems in Hilbert Space
}

\author{
Xisheng Dai ${ }^{1,2}$ and Feng Yang ${ }^{1}$ \\ ${ }^{1}$ School of Electrical and Information Engineering, Guangxi University of Science and Technology, Liuzhou 545006, China \\ ${ }^{2}$ School of Information and Electrical Engineering, Shandong University of Science and Technology, Qingdao 266510, China \\ Correspondence should be addressed to Xisheng Dai; mathdxs@163.com
}

Received 11 April 2013; Accepted 23 June 2013

Academic Editor: G. M. N’Guérékata

Copyright (c) 2013 X. Dai and F. Yang. This is an open access article distributed under the Creative Commons Attribution License, which permits unrestricted use, distribution, and reproduction in any medium, provided the original work is properly cited.

\begin{abstract}
This paper concerns the complete controllability of the impulsive stochastic integrodifferential systems in Hilbert space. Based on the semigroup theory and Burkholder-Davis-Gundy's inequality, sufficient conditions of the complete controllability for impulsive stochastic integro-differential systems are established by using the Banach fixed point theorem. An example for the stochastic wave equation with impulsive effects is presented to illustrate the utility of the proposed result.
\end{abstract}

\section{Introduction}

It is well known that controllability is one of the fundamental concepts and plays an important role in control theory and engineering. The problem which is about controllability of linear and nonlinear stochastic systems represented by SODE (stochastic ordinary differential equation) in finite dimensional space has been extensively studied (e.g., [1-4] and references therein). The controllability for infinite dimensional stochastic systems represented by SPDE (stochastic partial differential equation) is natural generalization of stochastic systems in finite dimensional space [5]. According to the literature, at least three types of infinite dimensional stochastic systems have been studied, that is, approximate, complete, and $S$-controllability [6], so the controllability research of the infinite dimensional stochastic systems is usually more complicated than that of the finite dimensional. For linear stochastic system, the controllability problem has been studied by some authors $[6,7]$, which is shown as the following SPDE:

$$
\begin{gathered}
d x(t)=[A x(t)+B u(t)] d t+\Sigma(t) d W(t), \quad t \in[0, T], \\
x(0)=x_{0} \in \mathscr{L}_{2}(\Omega, H),
\end{gathered}
$$

where $x_{0}$ is $\mathscr{F}_{0}$-measurable, $H$ is separable Hilbert space, $A$ is the infinitesimal generator of a strongly continuous semigroup $S(t)$ on $H, B \in \mathscr{L}(U, H), u(t)$ is feedback control, $W(t)$ is $Q$-Wiener process, and $\Sigma \in \mathscr{L}_{2}\left(Q^{1 / 2} E, H\right)$. For nonlinear stochastic systems in infinite dimensional space, there are also many results on the controllability theory (see [8-13]).

On the other hand, the impulsive effects exist widely in many evolution processes in which the states are changed abruptly at certain moments of time, involving fields such as finance, economics, mechanics, electronics, and telecommunications (see [14] and references of therein). Impulsive differential systems have emerged as an important area investigation in applied sciences, and many papers have been published about the controllability of impulsive differential systems both in finite and infinite dimensional space. Sakthivel et al. [15] established the sufficient conditions for approximate controllability of nonlinear impulsive differential systems by Schauder's fixed point theorem; Li et al. [16] investigated the complete controllability of the first-order impulsive functional differential systems in Banach space using Schaefer's fixed point theorem; Chang [17] studied the complete controllability of impulsive functional differential systems with infinite delay; Sakthivel et al. [18] discussed complete controllability of second-order nonlinear impulsive differential systems. However, the complete controllability problem of 
impulsive stochastic integro-differential systems has not been investigated in infinite dimensional space yet, to the best of our knowledge, although [19-22], respectively, investigated the controllability of impulsive stochastic control systems in finite dimensional space by using contraction mapping principle; and Subalakshmi and Balachandran [23] studied the approximate controllability of nonlinear stochastic impulsive systems in Hilbert spaces by using Nussbaum's fixed point theorem. Based on Banach fixed point theorem, the proposed work in this paper on the complete controllability of the integro-differential stochastic systems with impulsive effects in Hilbert spaces is new in the literature.

In this paper, our main purpose is to show the complete controllability of following impulsive stochastic integrodifferential systems in Hilbert space,

$$
\begin{aligned}
& d x(t)=[A x(t)+B u(t) \\
& \left.\quad+F\left(t, x(t), \int_{0}^{t} f(t, s, x(s)) d s\right)\right] d t \\
& \quad+G\left(t, x(t), \int_{0}^{t} g(t, s, x(s)) d s\right) d w(t), \\
& \quad t \neq t_{k}, t \geqslant 0, \\
& \Delta x\left(t_{k}\right)=I_{k}\left(x_{t_{k}^{-}}\right), \quad t=t_{k}, k=1,2, \ldots, m, \\
& x(0)=x_{0} \in H,
\end{aligned}
$$

where $F:[0, T] \times H \times H \rightarrow H, G:[0, T] \times H \times H \rightarrow$ $\mathscr{L}_{2}\left(Q^{1 / 2} E, H\right), f, g:[0, T \times[0, T] \times H \rightarrow H$ are measurable mappings. $I_{k}\left(x_{t_{k}^{-}}\right)=x\left(t_{k}^{+}\right)-x\left(t_{k}^{-}\right), t=t_{k}, k=1,2, \ldots$, $\rho$, where $x\left(t_{k}^{+}\right)$and $x\left(t_{k}^{-}\right)$denote the right and left limits of $x(t)$ at $t=t_{k}$, respectively. Also $\Delta x\left(t_{k}\right)=x\left(t_{k}^{+}\right)-x\left(t_{k}^{-}\right)$ represents the jump in the state $x$ at time $t_{k}$ with $I_{k}$ determining the size of the jump. For systems (2), if $I_{k}=0$, the controllability problem was studied by Subalakshmi et al. [11]. If $I_{k} \neq 0$ and $G=0, f=0,[15]$ discussed the approximate controllability problem. When $A, B$ are matrices with appropriate dimensions, $F, G$ are vectors (in fact, matrix is aspecial form of operator), and $f=g=0$, Karthikeyan et al. [19] obtained the controllability results, so system (2) is of the more general form and has great diversity.

The outline of this paper is as follows: Section 2 contains basic notations, lemmas, and preliminary facts. The controllability results are given in Section 3 by fixed point methods. In Section 4, we provide an example to demonstrate the effectiveness of our method. Finally, conclusions are given in Section 5.

\section{Preliminaries}

Let $(\Omega, \mathscr{F}, \mathbb{P})$ be a complete probability space with a filtration $\left\{\mathscr{F}_{t}\right\}_{t \geqslant 0}$ satisfying the usual conditions (i.e., it is right continuous and $\mathscr{F}_{0}$ contains all $\mathbb{P}$-null sets). We consider three Hilbert spaces $E, H$, and $U$, and a $Q$-Wiener process on $(\Omega, \mathscr{F}, \mathbb{P})$ with the covariance operator $Q \in \mathscr{L}(E)$ such that $\operatorname{tr} Q<\infty$. Let $\langle\cdot\rangle$ and $\|\cdot\|$ denote inner product and norm of $H$, respectively. $\mathscr{L}(X, Y)$ is the space of all linear bounded operator from a Hilbert space $X$ to a Hilbert space $Y$. We also employ the same notation $\|\cdot\|$ for the norm of $\mathscr{L}(X, Y)$. We assume that there exists a complete orthonormal $\left\{e_{k}\right\}$ in $E$, a bounded sequence of nonnegative real numbers $\lambda_{k}$ such that $Q e_{k}=\lambda_{k} e_{k}, k=1,2, \ldots$, and a sequence $\left\{\beta_{k}\right\}$ of independent Brownian motions such that

$$
\langle w(t), e\rangle=\sum_{k=1}^{\infty} \sqrt{\lambda_{k}}\left\langle e_{k}, e\right\rangle \beta_{k}(t), \quad e \in E, t \in[0, T],
$$

and $\mathscr{F}_{t}=\mathscr{F}_{t}^{\beta}$, where $\mathscr{F}_{t}^{\beta}$ is the $\sigma$-algebra generated by $\{\beta(s)$ : $0 \leqslant s \leqslant t\}$. Let $\mathscr{L}_{2}^{0}=\mathscr{L}_{2}\left(Q^{1 / 2} E, H\right)$ be the space of all HilbertSchmidt operator from $Q^{1 / 2} E$ to $H$ with the inner product $\langle\Psi, \Phi\rangle_{\mathscr{L}_{2}^{0}}=\operatorname{tr}\left[\Psi Q \Phi^{*}\right]$ and the norm $\|\cdot\|_{\mathscr{L}_{2}^{0}} \cdot L_{2}\left(\mathscr{F}_{T}, H\right)$ is the Hilbert space of all $\mathscr{F}_{T}$-measurable square integrable random variables with values in Hilbert space $H . L_{2}^{\mathscr{F}_{T}}([0, T], H)$ is the Hilbert space of square integrable and $\mathscr{F}_{T}$-adapted processes with values in $H$.

Let $\operatorname{PC}\left([0, T], L_{2}(\Omega, \mathscr{F}, \mathbb{P} ; H)\right)=\{\phi: \phi$ is a function from $[0, T]$ into $L_{2}(\Omega, \mathscr{F}, \mathbb{P} ; H)$ such that $\phi(t)$ is continuous at $t \neq t_{k}$, left continuous at $t=t_{k}$, and the right limit $\phi\left(t_{k}^{+}\right)$exists for $\left.k=1,2, \ldots, \rho\right\} . \mathscr{H}_{2}\left(U_{2}\right)$ is the closed subspace of $\mathrm{PC}\left([0, T], L_{2}(\Omega, \mathscr{F}, \mathbb{P} ; H)\right)$ consisting of measurable and $\mathscr{F}_{t}$-adapted $H$-valued( $U$-valued) process $\phi(\cdot) \quad \epsilon$ $\operatorname{PC}\left([0, T], L_{2}(\Omega, \mathscr{F}, \mathbb{P} ; H)\right)\left(\phi(\cdot) \in \operatorname{PC}\left([0, T], L_{2}(\Omega, \mathscr{F}, \mathbb{P} ; U)\right)\right)$ endowed with the norm $\|\phi\|_{\mathscr{H}_{2}}^{2}=\sup _{0 \leqslant t \leqslant T} \mathbb{E}\|\phi(t)\|^{2}$.

By a solution of system (2), we mean a mild solution of the following nonlinear integral equation:

$$
\begin{aligned}
x(t)= & S(t) x_{0}+\int_{0}^{t} S(t-s) B u(s) d s \\
& +\int_{0}^{t} S(t-s) F\left(s, x(s), \int_{0}^{s} f(s, \tau, x(\tau)) d \tau\right) d s \\
& +\int_{0}^{t} S(t-s) G\left(s, x(s), \int_{0}^{s} g(s, \tau, x(\tau)) d \tau\right) d w(s) \\
& +\sum_{k=1}^{\rho} S\left(t-t_{k}\right) I_{k}\left(x\left(t_{k}^{-}\right)\right),
\end{aligned}
$$

where $u \in U_{a d}:=U_{2}, S(t)_{t \geqslant 0}$ denotes the strongly continuous semigroup generated by the operator $A$.

Now let us introduce the controllability operator $\Pi_{s}^{T}$ associated with (4) (see[8]),

$$
\Pi_{s}^{T}\{\cdot\}=\int_{s}^{T} S(T-t) B B^{*} S^{*}(T-t) d t
$$

which belongs to $\mathscr{L}(H, H) ; B^{*}$ is the adjoint operator of $B$.

Definition 1. System (2) is completely controllable on $[0, T]$ if

$$
\mathscr{R}_{T}\left(x_{0}\right)=L_{2}^{\mathscr{F}_{T}}([0, T], H) .
$$


That is, all the points in $L_{2}^{\mathscr{F}_{T}}([0, T], H)$ can be reached from the point $x_{0}$ at time $T$, where $\mathscr{R}_{t}\left(x_{0}\right)=\left\{x\left(t ; x_{0}, u\right): u \in\right.$ $\left.L_{2}^{\mathscr{F}}([0, T], H)\right\}$.

Lemma 2 (Burkholder-Davis-Gundy's inequality [23]). For any $r \geqslant 1$ and for arbitrary $\mathscr{L}_{2}^{0}$-valued predictable process $\Psi(t)$, $t \in[0, T]$, one has

$$
\mathbb{E}\left(\sup _{0 \leqslant t \leqslant T}\left|\int_{0}^{t} \Psi(s) d w(s)\right|^{2 r}\right) \leqslant C_{r} \mathbb{E}\left(\int_{0}^{t}\|\Psi(s)\|_{\mathscr{L}_{2}^{0}}^{2} d s\right)^{r},
$$

where

$$
C_{r}=(r(2 r-1))^{r}\left(\frac{2 r}{2 r-1}\right)^{2 r^{2}} .
$$

Lemma 3 (Mahmudov [6]). The following linear system

$$
d x(t)=[A x(t)+B u(t)] d t+D(t) d w(t), \quad x(0)=x_{0}
$$

is completely controllable if and only if $\Pi_{0}^{T} \geqslant \gamma I$, where $\gamma$ is constant and I is unit operator.

Lemma 4. Assume that the operator $\Pi_{s}^{T}$ is invertible. Then for arbitrary target $x_{T} \in L_{2}\left(\mathscr{F}_{T}, H\right)$, the control

$$
\begin{aligned}
u(t)= & B^{*} S^{*}(T-t) \mathbb{E} \\
& \times\left\{( \Pi _ { 0 } ^ { T } ) ^ { - 1 } \left[x_{T}-S(T) x_{0}-\int_{0}^{T} S(T-s) \bar{F}(s) d s\right.\right. \\
& -\int_{0}^{T} S(T-s) \bar{G}(s) d w(s) \\
& \left.\left.-\sum_{k=1}^{\rho} S\left(T-t_{k}\right) I_{k}\left(x\left(t_{k}^{-}\right)\right)\right] \mid \mathscr{F}_{t}\right\}
\end{aligned}
$$

transfers the systems (4) from $x_{0}$ to $x_{T}$ at time $T$, where $\bar{F}(s)=$ $F\left(s, x(s), \int_{0}^{s} f(s, \tau, x(\tau)) d \tau\right), \bar{G}(s)=G\left(s, x(s), \int_{0}^{s} g(s, \tau, x(\tau))\right.$ $d \tau)$.

Proof. Substituting (10) into (4), we can obtain that

$$
\begin{aligned}
x(t)= & S(t) x_{0}+\int_{0}^{t} S(t-s) \\
& \times B B^{*} S^{*}(T-s) \mathbb{E} \\
& \times\left\{( \Pi _ { 0 } ^ { T } ) ^ { - 1 } \left[x_{T}-S(T) x_{0}-\int_{0}^{T} S(T-s) \bar{F}(s) d s\right.\right. \\
& -\int_{0}^{T} S(T-s) \bar{G}(s) d w(s) \\
& \left.\left.\quad-\sum_{k=1}^{\rho} S\left(T-t_{k}\right) I_{k}\left(x\left(t_{k}^{-}\right)\right)\right] \mid \mathscr{F}_{t}\right\}
\end{aligned}
$$

$$
\begin{aligned}
& +\int_{0}^{t} S(T-s) \bar{F}(s) d s \\
& +\int_{0}^{t} S(T-s) \bar{G}(s) d w(s)+\sum_{k=1}^{\rho} S\left(T-t_{k}\right) I_{k}\left(x\left(t_{k}^{-}\right)\right) \\
& =S(t) x_{0}+\Pi_{0}^{t}\left\{S^{*}(T-t)\left(\Pi_{0}^{T}\right)^{-1}\right. \\
& \quad \times\left[x_{T}-S(T) x_{0}-\int_{0}^{T} S(T-s) \bar{F}(s) d s\right. \\
& \quad-\int_{0}^{t} S(t-s) \bar{G}(s) d w(s) \\
& \left.\left.+\sum_{k=1}^{\rho} S\left(T-t_{k}\right) I_{k}\left(x\left(t_{k}^{-}\right)\right)\right]\right\} \\
& +\sum_{k=1}^{\rho} S\left(T-t_{k}\right) I_{k}\left(x\left(t_{k}^{-}\right)\right) . \\
& +\int_{0}^{t} S(s) d s+\int_{0}^{t} S(T-s) \bar{G}(s) d w(s)
\end{aligned}
$$

The proof is completed by letting $t=T$ in (11).

\section{Main Results}

In this section, by using contraction mapping principle in Banach space we discuss the complete controllability criteria of semilinear impulsive stochastic systems (2). For the proof of the main result we impose the following assumptions on data of the problem.

Assumption A. The functions $F, G$, and $I$ are continuous and satisfy the usual linear growth condition; that is, there exist positive real constants $L_{1}, \alpha_{k}$ for arbitrary $x \in H$, and $0 \leqslant$ $t \leqslant T$ such that

$$
\begin{gathered}
\|F(t, x, y)\|^{2}+\|G(t, x, y)\|_{\mathcal{L}_{2}^{0}}^{2} \leqslant L_{1}\left(1+\|x\|^{2}+\|y\|^{2}\right), \\
\left\|I_{k}(x)\right\|^{2} \leqslant \alpha_{k}\left(1+\|x\|^{2}\right), \quad k=1,2, \ldots, \rho, \\
\left\|\int_{0}^{t} f(t, s, x(s)) d s\right\|^{2}+\left\|\int_{0}^{t} g(t, s, x(s)) d s\right\|^{2} \leqslant k_{1}\|x\|^{2} .
\end{gathered}
$$

Assumption $B$. The functions $F, G$, and $I$ satisfy the following Lipschitz condition and for every $t \geqslant 0$ and $x, y \in H$ there exist positive real constants $L_{2}, \beta_{k}, k_{2}$ such that

$$
\begin{gathered}
\left\|F\left(t, x_{1}, y_{1}\right)-F\left(t, x_{2}, y_{2}\right)\right\|^{2} \\
+\left\|G\left(t, x_{1}, y_{1}\right)-G\left(t, x_{2}, y_{2}\right)\right\|_{\mathscr{L}_{2}^{0}}^{2} \\
\leqslant L_{2}\left(\left\|x_{1}-x_{2}\right\|^{2}+\left\|y_{1}-y_{2}\right\|^{2}\right), \\
\left\|I_{k}(x)-I_{k}(y)\right\| \leqslant \beta_{k}\|x-y\|, \quad k=1,2, \ldots, \rho,
\end{gathered}
$$




$$
\begin{aligned}
& \int_{0}^{t}\left\{\|f(t, s, x(s))-f(t, s, y(s))\|^{2}\right. \\
& \left.\quad+\int_{0}^{t}\|g(t, s, x(s))-g(t, s, y(s))\|^{2}\right\} d s \\
& \quad \leqslant k_{2}\|x-y\|^{2} .
\end{aligned}
$$

Assumption C. The linear system (9) is completely controllable. By Lemma 3, for some $\gamma>0, \mathbb{E}\left\langle\Pi_{0}^{T} z, z\right\rangle \geqslant \gamma \mathbb{E}\|z\|^{2}$, for all $z \in L_{2}\left(\mathscr{F}_{T}, H\right)$. Consequently, $\left\|\left(\Pi_{0}^{T}\right)^{-1}\right\| \leqslant 1 / \gamma=l_{2}$.

Assumption D. Let $p=\left[6 T l_{1} L_{2}\left(M l_{1} l_{2}+1\right)(T+4)\left(1+k_{2} T\right)+\right.$ $\left.6 l_{1} \rho\left(M l_{2}+1\right) \sum_{k=1}^{\rho} \beta_{k}\right]$ be such that $0 \leqslant p<1$.

Now for convenience, let us introduce the following notations:

$$
l_{1}=\max _{0 \leqslant t \leqslant T}\|S(t)\|^{2}, \quad M=\max _{s \in[0, T]}\left\|\Pi_{s}^{T}\right\|^{2} .
$$

Theorem 5. Suppose that assumptions A, B, C, and D are satisfied. Then system (4) is completely controllable on $[0, T]$.

Proof. For arbitrary initial data $x_{0} \in \mathscr{H}_{2}$, we can define a nonlinear operator $\Phi$ from $\mathrm{H}_{2}$ to $\mathrm{H}_{2}$ as the following:

$$
\begin{aligned}
(\Phi x)(t)= & S(t) x_{0}+\int_{0}^{t} S(t-s) B u(s) d s \\
& +\int_{0}^{t} S(t-s) \bar{F}(s, x) d s \\
& +\int_{0}^{t} S(t-s) \bar{G}(s, x) d w(s) \\
& +\sum_{k=1}^{\rho} S\left(t-t_{k}\right) I_{k}\left(x\left(t_{k}^{-}\right)\right),
\end{aligned}
$$

where $u(t)$ is defined by (10).

By Lemma 4, the control (10) transfers system (4) from the initial state $x_{0}$ to the final state $x_{T}$ provided that the operators $\Phi$ has a fixed point in $\mathscr{H}_{2}$. So, if the operator $\Phi$ has a fixed point then system (2) is completely controllable. As mentioned before, to prove the complete controllability of the system (2), it is enough to show that $\Phi$ has a fixed point in $\mathscr{H}_{2}$. To do this, we can employ the contraction mapping principle. In the following, we will divide the proof into two steps.

Firstly, we show that $\mathscr{H}_{2}$ maps $\mathscr{H}_{2}$ into itself. From (15) we have

$$
\begin{aligned}
\sup _{0 \leqslant t \leqslant T} \mathbb{E}\|(\Phi x)(t)\|^{2}= & \sup _{0 \leqslant t \leqslant T} \mathbb{E} \| S(t) x_{0}+\int_{0}^{t} S(t-s) B u(s) d s \\
& +\int_{0}^{t} S(t-s) \bar{F}(s, x) d s \\
& +\int_{0}^{t} S(t-s) \bar{G}(s, x) d w(s)
\end{aligned}
$$

$$
\begin{aligned}
& +\sum_{k=1}^{\rho} S\left(t-t_{k}\right) I_{k}\left(x\left(t_{k}^{-}\right)\right) \|^{2} \\
& \qquad 5 \sup _{0 \leqslant t \leqslant T} \mathbb{E}\left\|S(t) x_{0}\right\|^{2} \\
& +\underset{0 \leqslant t \leqslant T}{ } \sup \mathbb{E}\left\|\int_{0}^{t} S(t-s) B u(s) d s\right\|^{2} \\
& +5 \sup _{0 \leqslant t \leqslant T} \mathbb{E}\left\|\int_{0}^{t} S(t-s) \bar{F}(s, x) d s\right\|^{2} \\
& +5 \sup _{0 \leqslant t \leqslant T} \mathbb{E}\left\|\int_{0}^{t} S(t-s) \bar{G}(s, x) d w(s)\right\|^{2} \\
& +5 \sup _{0 \leqslant t \leqslant T} \mathbb{E}\left\|\sum_{k=1}^{\rho} S\left(t-t_{k}\right) I_{k}\left(x\left(t_{k}^{-}\right)\right)\right\|^{2} \\
& \triangleq \sum_{i=1}^{5} A_{i} .
\end{aligned}
$$

Using Holder inequality, B-D-G inequality (here $C_{1}=4$ ), and Assumption $\mathrm{C}$, we have the following estimates:

$$
\begin{aligned}
& A_{1} \leqslant 5 l_{1} \mathbb{E}\left\|x_{0}\right\|^{2} \\
& A_{3} \leqslant 5 T \sup _{0 \leqslant t \leqslant T} \mathbb{E} \int_{0}^{t}\|S(t-s) \bar{F}(s, x)\|^{2} d s \\
& \leqslant 5 T l_{1} L_{1} \int_{0}^{T}\left(1+\left(1+k_{1}\right) \sup _{0 \leqslant s \leqslant T} \mathbb{E}\|x(s)\|^{2}\right) d s, \\
& A_{4} \leqslant 20 l_{1} L_{1} \int_{0}^{T}\left(1+\left(1+k_{1}\right) \sup _{0 \leqslant s \leqslant T} \mathbb{E}\|x(s)\|^{2}\right) d s, \\
& A_{5} \leqslant 5 \rho \sup _{0 \leqslant t \leqslant T} \mathbb{E} \sum_{k=1}^{\rho}\left\|S\left(t-t_{k}\right) I_{k}\left(x\left(t_{k}^{-}\right)\right)\right\|^{2} \\
& \quad \leqslant 5 \rho l_{1} \sum_{i-1}^{\rho} \alpha_{k}\left(1+\sup _{0 \leqslant t \leqslant T} \mathbb{E}\|x(s)\|^{2}\right) .
\end{aligned}
$$

Meanwhile by control function (15), we have

$$
\begin{aligned}
& A_{2}=5 \sup _{0 \leqslant t \leqslant T} \mathbb{E} \\
& \quad \times \| \int_{0}^{t} S(t-r) B B^{*} S^{*}(T-r) \mathbb{E} \\
& \quad \times\left\{\left(\Pi_{0}^{T}\right)^{-1} \times\left[x(T)-S(T) x_{0}\right.\right. \\
& \quad-\int_{0}^{T} S(T-s) \bar{F}(s, x) d s
\end{aligned}
$$




$$
\begin{gathered}
-\int_{0}^{T} S(T-s) \bar{G}(s, x) d w(s) \\
\left.\left.-\sum_{k=1}^{\rho} S\left(T-t_{k}\right) I_{k}\left(x\left(t_{k}^{-}\right)\right)\right]\right\} d r \|^{2} \\
=5 \sup _{0 \leqslant t \leqslant T} \mathbb{E} \| \int_{0}^{t} S(t-r) B B^{*} S^{*}(t-r) S^{*}(T-t)\left(\Pi_{0}^{T}\right)^{-1} \\
\times\left[x(T)-S(T) x_{0}-\int_{0}^{T} S(T-s) \bar{F}(s, x) d s\right. \\
-\int_{0}^{T} S(T-s) \bar{G}(s, x) d w(s) \\
\left.-\sum_{k=1}^{\rho} S\left(T-t_{k}\right) I_{k}\left(x\left(t_{k}^{-}\right)\right)\right] d r \|^{2} \\
=5 \sup _{0 \leqslant t \leqslant T} \mathbb{E} \Pi_{0}^{t} S^{*}(T-t)\left(\Pi_{0}^{T}\right)^{-1} \\
\times\left[x_{T}-S(T) x_{0}-\int_{0}^{T} S(T-s) \bar{F}(s) d s\right. \\
-\int_{0}^{t} S(t-s) \bar{G}(s, x) d w(s) \\
\left.-\sum_{k=1}^{\rho} S\left(T-t_{k}\right) I_{k}\left(x\left(t_{k}^{-}\right)\right)\right] \|^{2} .
\end{gathered}
$$

So similar as in (17), we get

$$
\begin{aligned}
A_{2} \leqslant 25 M l_{1} l_{2}(\mathbb{E} & \left\|x_{T}\right\|^{2}+l_{1}\left\|x_{0}\right\|^{2} \\
& +T l_{1} L_{1} \int_{0}^{T}\left(1+\left(1+k_{1}\right) \sup _{0 \leqslant s \leqslant T}\|x(s)\|^{2}\right) d s \\
& +4 l_{1} L_{1} \int_{0}^{T}\left(1+\left(1+k_{1}\right) \sup _{0 \leqslant s \leqslant T}\|x(s)\|^{2}\right) d s \\
& \left.+\rho \sum_{i=1}^{\rho} \alpha_{k}\left(1+\sup _{0 \leqslant t \leqslant T}\|x(t)\|^{2}\right)\right) .
\end{aligned}
$$

From (17)-(19), we have

$$
\begin{array}{rl}
\sup _{0 \leqslant t \leqslant T} & \mathbb{E}\|\Phi x(t)\|^{2} \\
& \leqslant C\left[1+\int_{0}^{T}\left(1+\left(1+k_{1}\right) \sup _{0 \leqslant s \leqslant T} \mathbb{E}\|x(s)\|^{2}\right) d s\right] \\
& \leqslant C\left[1+\left(1+\left(1+k_{1}\right) T \sup _{0 \leqslant s \leqslant T} \mathbb{E}\|x(s)\|^{2}\right)\right]
\end{array}
$$

for all $t \in[0, T]$, where $C$ is constant. This implies that $\Phi$ maps $\mathscr{H}_{2}$ into itself.

Secondly, we prove that $\Phi$ is a contraction mapping on $\mathscr{H}_{2}$, for any $x, y \in \mathscr{H}_{2}$,

$$
\begin{aligned}
& \sup _{0 \leqslant t \leqslant T} \mathbb{E}\|(\Phi x)(t)-(\Phi y)(t)\|^{2} \\
& =\sup _{0 \leqslant t \leqslant T} \mathbb{E} \| \Pi_{0}^{t} S^{*}(T-t)\left(\Pi_{0}^{T}\right)^{-1} \\
& \times\left[\int_{0}^{T} S(T-s)(\bar{F}(s, y)-\bar{F}(s, x)) d s\right. \\
& +\int_{0}^{T} S(T-s)(\bar{G}(s, y)-\bar{G}(s, x)) d w(s) \\
& \left.+\sum_{k=1}^{\rho} S\left(T-t_{k}\right)\left(I_{k}\left(y\left(t_{k}^{-}\right)\right)-I_{k}\left(x\left(t_{k}^{-}\right)\right)\right)\right] \\
& +\int_{0}^{t} S(t-s)(\bar{F}(s, x)-\bar{F}(s, y)) d s \\
& +\int_{0}^{t} S(t-s)(\bar{G}(s, x)-\bar{G}(s, y)) d w(s) \\
& +\sum_{k=1}^{\rho} S\left(t-t_{k}\right)\left(I_{k}\left(x\left(t_{k}^{-}\right)\right)-I_{k}\left(y\left(t_{k}^{-}\right)\right)\right) \|^{2} \\
& \leqslant 6 M l_{1} l_{2}\left\{\left\|\int_{0}^{T} S(T-s)(\bar{F}(s, x)-\bar{F}(s, y)) d s\right\|^{2}\right. \\
& +\left\|\int_{0}^{T} S(T-s)(\bar{G}(s, x)-\bar{G}(s, y)) d s\right\|^{2} \\
& \left.+\left\|\sum_{k=1}^{\rho} S\left(T-t_{k}\right)\left(I_{k}\left(x\left(t_{k}^{-}\right)\right)-I_{k}\left(y\left(t_{k}^{-}\right)\right)\right)\right\|^{2}\right\} \\
& +6 \sup _{0 \leqslant t \leqslant T} \mathbb{E}\left\|\int_{0}^{t} S(t-s)(\bar{F}(s, x)-\bar{F}(s, y)) d s\right\|^{2} \\
& +6 \sup _{0 \leqslant t \leqslant T} \mathbb{E}\left\|\int_{0}^{t} S(t-s)(\bar{G}(s, x)-\bar{G}(s, y)) d w(s)\right\|^{2} \\
& +6 \sup _{0 \leqslant t \leqslant T} \mathbb{E}\left\|\sum_{k=1}^{\rho} S\left(t-t_{k}\right)\left(I_{k}\left(x\left(t_{k}^{-}\right)\right)-I_{k}\left(y\left(t_{k}^{-}\right)\right)\right)\right\|^{2} \\
& \triangleq 6 M l_{1} l_{2} \sum_{i=1}^{3} B_{i}+\sum_{i=4}^{6} B_{i}
\end{aligned}
$$

Using Lipschitz condition, similiar to $A_{1}-A_{5}$, we have the following estimates:

$$
B_{1} \leqslant T l_{1} L_{2}\left(1+k_{2} T\right) \int_{0}^{T} \sup _{0 \leqslant s \leqslant T} \mathbb{E}\|x(s)-y(s)\|^{2} d s,
$$




$$
\begin{aligned}
& B_{2} \leqslant 4 l_{1} L_{2}\left(1+k_{2} T\right) \int_{0}^{T} \sup _{0 \leqslant s \leqslant T} \mathbb{E}\|x(s)-y(s)\|^{2} d s, \\
& B_{3} \leqslant l_{1} \rho \sum_{k=1}^{\rho} \beta_{k} \sup _{0 \leqslant s \leqslant T} \mathbb{E}\|x(s)-y(s)\|^{2}, \\
& B_{4} \leqslant 6 T l_{1} L_{2}\left(1+k_{2} T\right) \int_{0}^{T} \sup _{0 \leqslant s \leqslant T} \mathbb{E}\|x(s)-y(s)\|^{2} d s, \\
& B_{5} \leqslant 24 l_{1} L_{2}\left(1+k_{2} T\right) \int_{0}^{T} \sup _{0 \leqslant s \leqslant T} \mathbb{E}\|x(s)-y(s)\|^{2} d s, \\
& B_{6} \leqslant 6 l_{1} \rho \sum_{k=1}^{\rho} \beta_{k} \sup _{0 \leqslant s \leqslant T} \mathbb{E}\|x(s)-y(s)\|^{2}
\end{aligned}
$$

together with inequalities (22)-(27):

$$
\begin{aligned}
\sup _{0 \leqslant t \leqslant T} \mathbb{E} & \|(\Phi x)(t)-(\Phi y)(t)\|^{2} \\
\leqslant & 6 T M l_{1}^{2} l_{2} L_{2}\left(1+k_{2} T\right) \int_{0}^{T} \sup _{0 \leqslant s \leqslant T} \mathbb{E}\|x(s)-y(s)\|^{2} d s \\
& +24 M l_{1}^{2} l_{2} L_{2}\left(1+k_{2} T\right) \int_{0}^{T} \sup _{0 \leqslant s \leqslant T} \mathbb{E}\|x(s)-y(s)\|^{2} d s \\
& +\left(6 M l_{1} l_{2} \rho \sum_{k=1}^{\rho} \beta_{k}+6 l_{1} \rho \sum_{k=1}^{\rho} \beta_{k}\right) \sup _{0 \leqslant t \leqslant T} \mathbb{E}\|x(t)-y(t)\|^{2} \\
\leqslant & {\left[6 T l_{1} L_{2}\left(M l_{1} l_{2}+1\right)(T+4)\left(1+k_{2} T\right)\right.} \\
& \left.+6 l_{1} \rho\left(M l_{2}+1\right) \sum_{k=1}^{\rho} \beta_{k}\right] \sup _{0 \leqslant t \leqslant T} \mathbb{E}\|x(t)-y(t)\|^{2} .
\end{aligned}
$$

Theorefore, $\Phi$ is a contraction mapping from $\mathscr{H}_{2}$ to $\mathscr{H}_{2}$, and hence $\Phi$ has a unique fixed point. Thus the system (4) is completely controllability on $[0, T]$.

\section{Example}

Consider the impulsive stochastic integrao-differential wave equation with control $u(t, z) \in L^{2}[0,1]$,

$$
\begin{gathered}
\frac{\partial^{2} z}{\partial t^{2}}=a \frac{\partial^{2} z}{\partial \xi^{2}}+b u(t, z)+F\left(t, z(t), \int_{0}^{t} f(t, s, z(s)) d s\right) \\
+G\left(t, z(t), \int_{0}^{t} g(t, s, z(s)) d s\right) \frac{\partial w}{\partial t}, \\
t \neq t_{k}, 0 \leqslant \xi \leqslant 1,
\end{gathered}
$$

where $\partial w / \partial t$ is white noise and initial and boundary conditions are

$$
\begin{gathered}
z(t, 0)=z(t, 1)=0, \quad t \neq t_{k}, \\
\Delta z\left(t_{k}\right)(\xi)=I_{k}^{1}\left(z_{t_{k}}\right), \quad \Delta z^{\prime}\left(t_{k}\right)(\xi)=I_{k}^{2}\left(z_{t_{k}}\right), \\
z(0, \xi)=z_{0}(\xi), \quad \frac{\partial z(0, \xi)}{\partial t}=z_{1}(\xi) .
\end{gathered}
$$

Let $H=L^{2}[0,1]$; then $A: H \rightarrow H, A z=z^{\prime \prime}$. Domain of operator $A$ is

$$
\begin{gathered}
D(A)=\left\{z \in H \mid z, z^{\prime} \text { is continuous, } z^{\prime \prime} \in H,\right. \\
z(0)=z(1)=0\} .
\end{gathered}
$$

Let

$$
\begin{gathered}
Z=\left[\begin{array}{c}
z \\
\frac{\partial z}{\partial t}
\end{array}\right], \quad Z(0)=\left[\begin{array}{l}
z_{0} \\
z_{1}
\end{array}\right], \quad B=\left[\begin{array}{l}
0 \\
b
\end{array}\right], \\
\bar{F}=\left[F\left(t, z(t), \int_{0}^{t} f(t, s, z(s)) d s\right)\right], \\
\bar{G}=\left[G\left(t, z(t), \int_{0}^{t} g(t, s, z(s)) d s\right)\right] .
\end{gathered}
$$

Then the system (29) is

$$
\begin{gathered}
d Z=[\mathscr{A} Z+B u+\bar{F}(t, Z)] d t+\bar{G}(t, Z) d W, \quad t \neq t_{k}, \\
\Delta Z_{k}=\left[\begin{array}{c}
I_{k}^{1} \\
I_{k}^{2}
\end{array}\right], \quad t=t_{k}, k=1,2, \ldots, m, Z(0)=\left[\begin{array}{l}
z_{0} \\
z_{1}
\end{array}\right],
\end{gathered}
$$

where

$$
\mathscr{A}=\left[\begin{array}{cc}
0 & I \\
-A & 0
\end{array}\right]
$$

and $\mathscr{A}$ is the infinitesimal generator of a strongly continuous semigroup $h(t), t \geqslant 0$, on $X=D\left(A^{1 / 2}\right) \oplus H$, for $x \times y \in X$ :

$$
S(t)\left[\begin{array}{l}
x \\
y
\end{array}\right]=\sum_{n=1}^{\infty}\left[\begin{array}{cc}
\cos (n \pi t) & (n \pi)^{-1} \sin (n \pi t) \\
-(n \pi) \sin (n \pi t) & \cos (n \pi t)
\end{array}\right]\left[\begin{array}{l}
x_{n} \\
y_{n}
\end{array}\right] e_{n}
$$

$e_{n}(\theta)=\sqrt{2} \sin (n \pi \theta), \theta \in[0,1]$. Write $h(t)$ is

$$
h(t)=\left[\begin{array}{ll}
C(t) & S(t) \\
A S(t) & C(t)
\end{array}\right]
$$

where

$$
S(t) x:=\int_{0}^{t} C(s) x d s, \quad x \in H
$$


The mild solution of system (29) is

$$
\begin{aligned}
z(t)= & C(t) z_{0}+S(t) z_{1}+\int_{0}^{t} S(t-s)[B u(s)+f(s, z)] d s \\
& +\int_{0}^{t} S(t-s) g(s, z) d w_{s} \\
& +\sum_{0 \leqslant t_{k} \leqslant t} C\left(t-t_{k}\right) I_{k}^{1}\left(z_{t_{k}}\right)+\sum_{0 \leqslant t_{k} \leqslant t} S\left(t-t_{k}\right) I_{k}^{2}\left(z_{t_{k}}\right)
\end{aligned}
$$

by [7]; the stochastic linear system of (29) is complete controllable. Then from Theorem 5 one can easily prove system (29) is completely controllable, if the functions $F, G, f, g, I_{k}^{1}, I_{k}^{2}$ satisfy Lipschitz condition and linear growth condition.

\section{Conclusions}

The complete controllability of impulsive stochastic integrodifferential systems in Hilbert space has been investigated in this paper. Sufficient conditions of complete controllability for impulsive stochastic integro-differential systems are established by using the Banach fixed point theorem. An example illustrates the efficiency of proposed results.

\section{Acknowledgments}

This work is supported by National Natural Science Foundation of China under Grant nos. 61273126 and 61174078, PhD Start-up Fundation of Guangxi University of Science and Technology (no. 03081520), and Guangxi Higher Education Science Research Projection (no. 201203YB125).

\section{References}

[1] N. I. Mahmudov, "Controllability of linear stochastic systems," IEEE Transactions on Automatic Control, vol. 46, no. 5, pp. 724731, 2001.

[2] A. Arapostathis, R. K. George, and M. K. Ghosh, "On the controllability of a class of nonlinear stochastic systems," Systems \& Control Letters, vol. 44, no. 1, pp. 25-34, 2001.

[3] K. Balachandran and S. Karthikeyan, "Controllability of nonlinear Itô type stochastic integrodifferential systems," Journal of the Franklin Institute, vol. 345, no. 4, pp. 382-391, 2008.

[4] K. Balachandran and S. Karthikeyan, "Controllability of stochastic integrodifferential systems," International Journal of Control, vol. 80, no. 3, pp. 486-491, 2007.

[5] G. da Prato and J. Zabczyk, Stochastic Equations in Infinite Dimensions, vol. 44 of Encyclopedia of Mathematics and Its Applications, Cambridge University Press, Cambridge, UK, 1992.

[6] N. I. Mahmudov, "Controllability of linear stochastic systems in Hilbert spaces," Journal of Mathematical Analysis and Applications, vol. 259, no. 1, pp. 64-82, 2001.

[7] M. A. Dubov and B. S. Mordukhovich, "On controllability of infinite dimensional linear stochastic systems," in Proceedings of the 2nd IFAC Symposium on Stochastic Control, pp. 307-310, Vilnius, Lithuania, May 1986.

[8] N. I. Mahmudov, "Controllability of semilinear stochastic systems in Hilbert spaces," Journal of Mathematical Analysis and Applications, vol. 288, no. 1, pp. 197-211, 2003.
[9] J. P. Dauer and N. I. Mahmudov, "Controllability of stochastic semilinear functional differential equations in Hilbert spaces," Journal of Mathematical Analysis and Applications, vol. 290, no. 2, pp. 373-394, 2004.

[10] J. Y. Park, P. Balasubramaniam, and N. Kumaresan, "Controllability for neutral stochastic functional integrodifferential infinite delay systems in abstract space," Numerical Functional Analysis and Optimization, vol. 28, no. 11-12, pp. 1369-1386, 2007.

[11] R. Subalakshmi, K. Balachandran, and J. Y. Park, "Controllability of semilinear stochastic functional integrodifferential systems in Hilbert spaces," Nonlinear Analysis: Hybrid Systems, vol. 3, no. 1, pp. 39-50, 2009.

[12] K. Balachandran and J. P. Dauer, "Controllability of nonlinear systems in Banach spaces: a survey," Journal of Optimization Theory and Applications, vol. 115, no. 1, pp. 7-28, 2002.

[13] P. Balasubramaniam and J. P. Dauer, "Controllability of semilinear stochastic delay evolution equations in Hilbert spaces," International Journal of Mathematics and Mathematical Sciences, vol. 31, no. 3, pp. 157-166, 2002.

[14] V. Lakshmikantham, D. D. Baǐnov, and P. S. Simeonov, Theory of Impulsive Differential Equations, vol. 6 of Series in Modern Applied Mathematics, World Scientific, Singapore, 1989.

[15] R. Sakthivel, N. I. Mahmudov, and J. H. Kim, "Approximate controllability of nonlinear impulsive differential systems," Reports on Mathematical Physics, vol. 60, no. 1, pp. 85-96, 2007.

[16] M. Li, M. Wang, and F. Zhang, "Controllability of impulsive functional differential systems in Banach spaces," Chaos, Solitons and Fractals, vol. 29, no. 1, pp. 175-181, 2006.

[17] Y.-K. Chang, "Controllability of impulsive functional differential systems with infinite delay in Banach spaces," Chaos, Solitons and Fractals, vol. 33, no. 5, pp. 1601-1609, 2007.

[18] R. Sakthivel, N. I. Mahmudov, and J. H. Kim, "On controllability of second order nonlinear impulsive differential systems," Nonlinear Analysis: Theory, Methods \& Applications, vol. 71, no. 1-2, pp. 45-52, 2009.

[19] S. Karthikeyan and K. Balachandran, "Controllability of nonlinear stochastic neutral impulsive systems," Nonlinear Analysis: Hybrid Systems, vol. 3, no. 3, pp. 266-276, 2009.

[20] R. Sakthivel, N. I. Mahmudov, and S.-G. Lee, "Controllability of non-linear impulsive stochastic systems," International Journal of Control, vol. 82, no. 5, pp. 801-807, 2009.

[21] R. Sakthivel, "Controllability of nonlinear impulsive Itô type stochastic systems," International Journal of Applied Mathematics and Computer Science, vol. 19, no. 4, pp. 589-595, 2009.

[22] L. Shen, J. Shi, and J. Sun, "Complete controllability of impulsive stochastic integro-differential systems," Automatica, vol. 46, no. 6, pp. 1068-1073, 2010.

[23] R. Subalakshmi and K. Balachandran, "Approximate controllability of nonlinear stochastic impulsive integrodifferential systems in Hilbert spaces," Chaos, Solitons and Fractals, vol. 42, no. 4, pp. 2035-2046, 2009. 


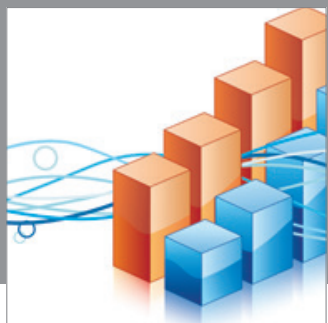

Advances in

Operations Research

mansans

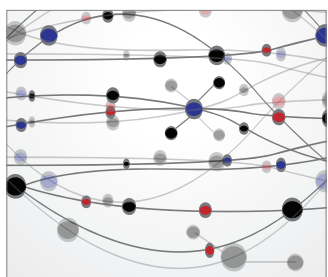

The Scientific World Journal
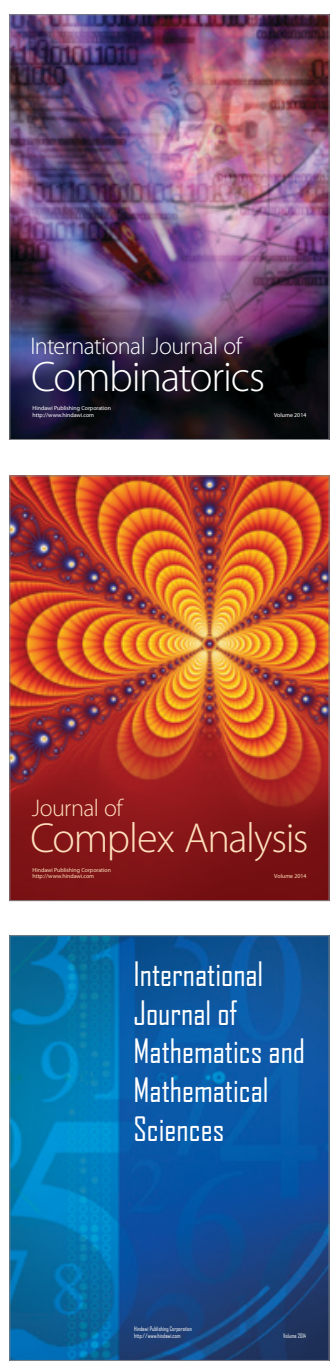
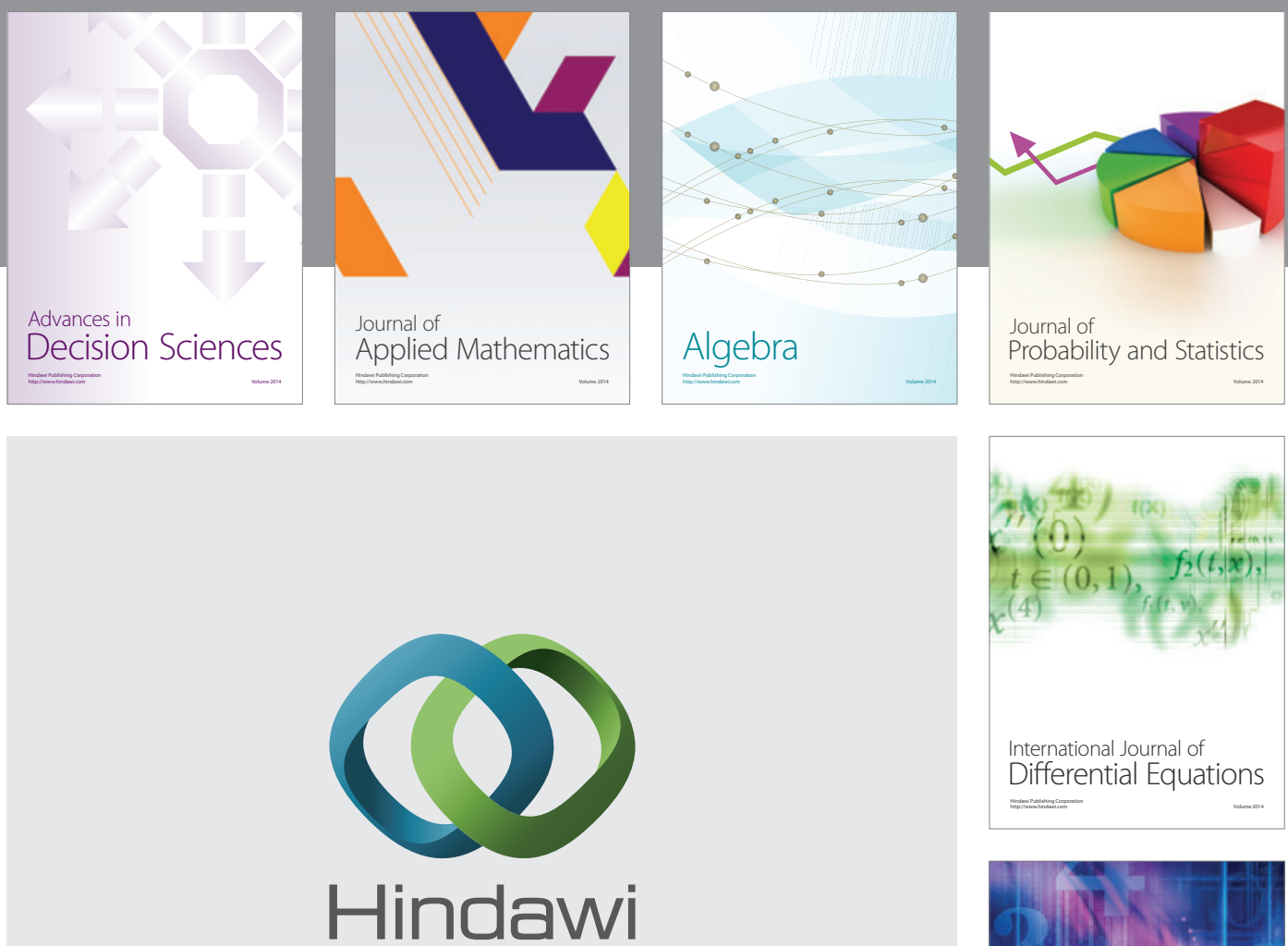

Submit your manuscripts at http://www.hindawi.com
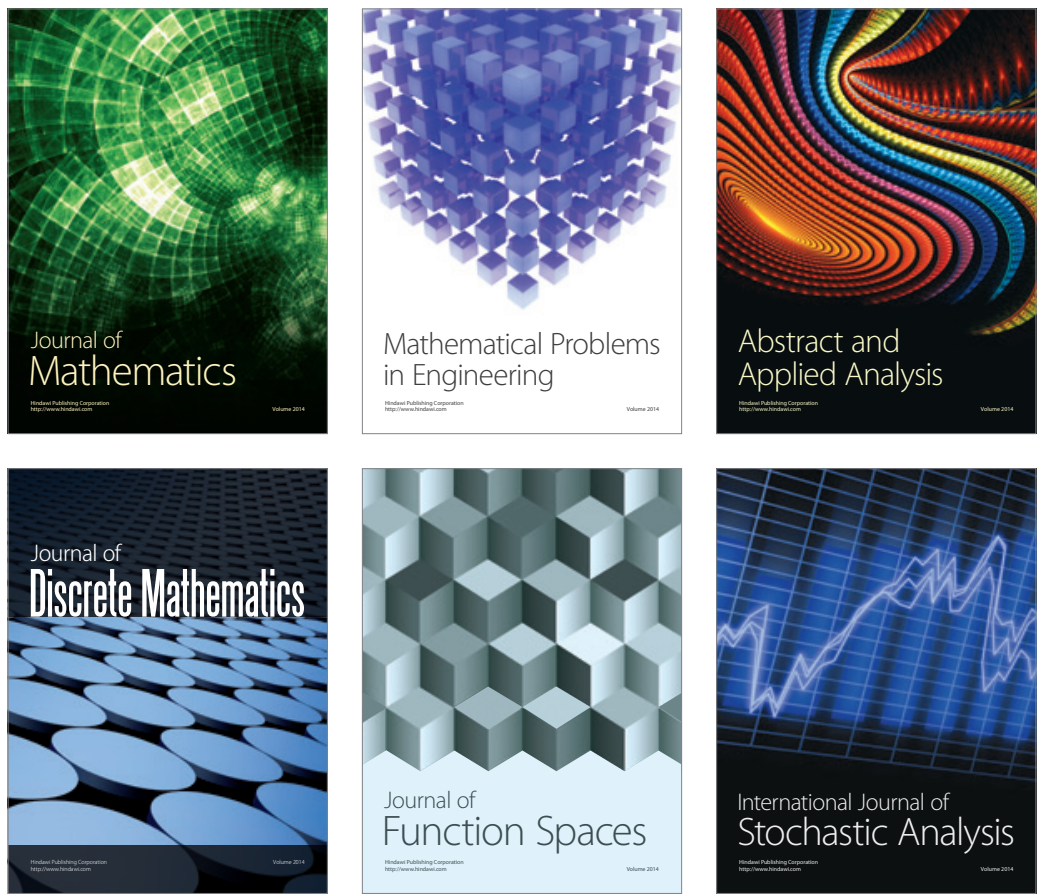

Journal of

Function Spaces

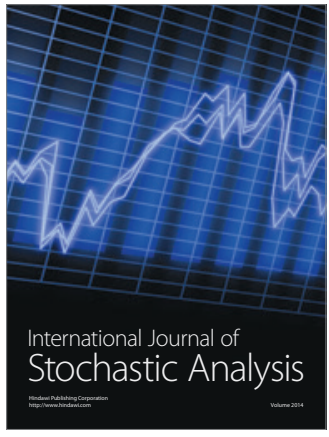

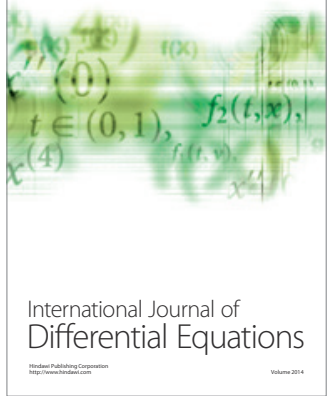
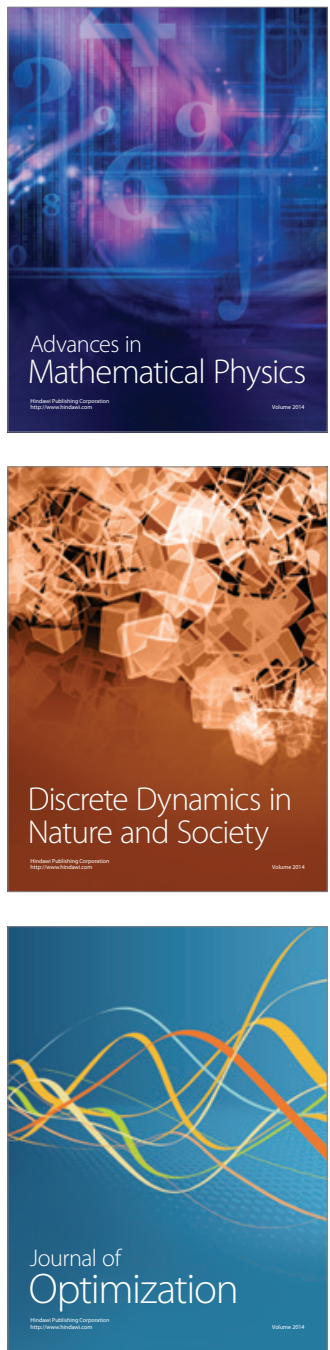